\title{
Entre routine conversationnelle et marqueur de discours : les usages de pardon dans certains français africains
}

\author{
Martina Drescher ${ }^{1}$ \\ Lehrstuhl für Romanische und Allgemeine Sprachwissenschaft, Universität Bayreuth, D-95447 \\ Bayreuth, Allemagne
}

\begin{abstract}
Résumé. La dynamique du français en Afrique touche non seulement son lexique et sa grammaire, mais aussi son dispositif énonciatif, ses modes d'organisation du discours et, de façon générale, son niveau pragmaticodiscursif. Partant de données recueillies au Cameroun et au Burkina Faso, l'étude se focalise sur les emplois interjectifs de pardon, qui semble évoluer d'une routine conversationnelle conversationnelle destinée à la gestion de l'interpersonnel vers un marqueur de discours avec des fonctions plus proprement discursives. Ces glissements dans le sens pragmatico-discursif de pardon vont de pair avec un élargissement de son champ fonctionnel. Équivalent de s'il vous plaît dans de nombreux contextes, il s'annexe ses valeurs d'emphase et de focalisation tout en contribuant à la structuration de l'énoncé. Sans prétendre à l'exhaustivité, la présente étude vise une première systématisation des fonctions discursives de pardon dans les français camerounais et burkinabé. Pour conclure, elle revient sur la question de savoir si ces modifications fonctionnelles de pardon sont dues à des interférences avec les langues de contact ou si elles renvoient plus globalement à des conventions de politesse et partant à une culture différente.
\end{abstract}

\begin{abstract}
Between conversational routine and discourse marker: Some uses of pardon in African varieties of French. The dynamics of French in Africa concerns not only its lexicon and its grammar, but also its enunciative and discourse organizational devices, and more generally, its pragmatic and discursive level. Based on data collected in Cameroon and in Burkina Faso, this study focuses on the interjectional uses of French pardon, that seems to evolve from a conversational routine maintaining interpersonal relations to a discourse marker fulfilling rather discursive functions. These shifts in the pragmatic and discursive meaning of pardon come along with an extension of its functions. Being an equivalent of $s^{\prime} i l$ vous plaît in numerous contexts, it annexes its emphasis and focalization qualities, but contributes also to the structuring of the utterance. Without pretending to be exhaustive, the present study aims at a first systematization of the discursive functions of pardon in Cameroonian and Burkinabe French. The conclusion comes back to the question whether
\end{abstract}

1

martina.drescher@uni-bayreuth.de 
these functional shifts are due to interferences with contact languages or whether they point more globally to different politeness and thus cultural conventions.

\section{Introduction}

Le français en Afrique connaît une grande dynamique. Les besoins identitaires et communicatifs de ses locuteurs ainsi que les langues co-présentes dans son milieu favorisent des processus d'appropriation qui se manifestent à travers l'émergence de formes et manières d'expression différentes de celles du français hexagonal (FH) (Drescher/Neumann-Holzschuh 2010). Si le lexique de ces 'nouveaux' français a fait l'objet de nombreuses études, il n'en est pas de même de leurs spécificités pragmaticodiscursives. Or, l'autonomisation croissante des français africains (FA) concerne aussi le dispositif énonciatif, les marqueurs de discours et, de façon générale, les modes d'organisation du discours, la réalisation des actes de langage ou des genres communicatifs. L'influence d'autres conventions discursives se manifeste également au niveau des « termes de civilité » (Grevisse ${ }^{10} 1975$ ) ou des routines conversationnelles comme merci, pardon, je vous en prie ou s'il vous plaît. Pour la plupart des unités polylexicales plus ou moins figées, certaines ne consistent cependant qu'en un seul mot. Typiques de l'oral, ces mots et locutions appartiennent aux phraséologismes pragmatiques qui se distinguent par leur lien stable avec un type de situation spécifique. Ils servent avant tout à la gestion des rapports sociaux tout en renseignant sur la relation interpersonnelle entre les interlocuteurs. Les routines conversationnelles se confondent parfois avec les marqueurs de discours avec lesquels elles partagent certaines fonctions. L'apport de ces derniers se situe cependant à un niveau plus proprement discursif. Ils contribuent à l'organisation et à la cohérence du discours, à son ancrage dans le contexte et à la coordination des interlocuteurs (Schiffrin 1996, 315). Certains ont des valeurs modales et expriment des attitudes affectives et / ou épistémiques. Constituant une classe fonctionnelle, les marqueurs sont hétérogènes du point de vue formel et regroupent adverbes, conjonctions, prépositions, interjections, phrases figées, etc.

Partant de données recueillies au Cameroun et au Burkina Faso, notre étude se focalise sur les emplois interjectifs de pardon. Nous partons de l'hypothèse que pardon participe aux processus d'appropriation qui caractérisent les FA en évoluant d'une routine conversationnelle limitée à la gestion de l'interpersonnel vers un marqueur de discours qui remplit des fonctions plus proprement discursives. Pardon subit des glissements dans son sens pragmatico-discursif qui vont de pair avec un élargissement de son champ fonctionnel. Équivalent de s'il vous plaît dans de nombreux contextes, il s'annexe ses valeurs d'emphase et de focalisation tout en contribuant à la structuration de l'énoncé. Sans prétendre à l'exhaustivité, nous visons une première systématisation des fonctions discursives de pardon dans les FA. Après une brève présentation de l'écologie du français en Afrique subsaharienne (chap. 2) et une discussion succincte des effets du contact linguistique sur les marqueurs de discours (chap. 3), nous passons à une première synthèse des usages de pardon à partir de son traitement dans des dictionnaires et grammaires (chap. 4). Suivront les analyses à partir d'extraits du corpus (chap. 5) et la conclusion (chap. 6).

\section{L'écologie du français en Afrique subsaharienne}

La dynamique du français en Afrique subsaharienne s'est accélérée avec les indépendances au début des années 1960. Une « écologie » différente comprenant «l'organisation sociale, la taille des groupes de locuteurs, les fonctions des langues, le rôle social de leurs locuteurs, leur degré de plurilinguisme, etc. » (Calvet 2000, 65) a conduit à une appropriation du 
français et à l'émergence de formes non-hexagonales. Le contact linguistique est le moteur principal de cette évolution qui aboutit à la genèse de normes endogènes. Au Cameroun, connu pour sa très grande diversité ethnolinguistique, on recense plus de 250 langues indigènes pour une population d'environ 23 millions d'habitants (Drescher 2017). Cette fragmentation linguistique, typique de la plupart des pays africains, conduit à des situations di- voire polyglossiques complexes où le français évolue dans un milieu caractérisé par la co-présence d'autres langues, très éloignées du français d'un point de vue typologique. De plus, le français reste encore majoritairement une langue seconde (L2) de sorte que dans la plupart des pays africains on a affaire à une "francophonie seconde » (Manessy 1994a). Une grande partie des apprenants n'arrive qu'à une maîtrise approximative qui se fossilise. Il en résulte une interlangue stabilisée qui porte les traces du contact linguistique emprunts et interférences - et qui est à la base de la norme endogène émergente. Par ailleurs, la créativité des locuteurs intervient dans l'adaptation d'une langue venue 'd'ailleurs' et guère apte à saisir les particularités des réalités africaines.

On peut faire l'hypothèse que les langues de contact africaines proposent une conceptualisation différente de l'expérience qui se reflète dans leur système linguistique. Manessy forge la notion de sémantaxe pour saisir la relation entre « des manières africaines de voir les choses et de catégoriser l'expérience » (Manessy 1994b, 89) et des structures linguistico-cognitives. Ces différences concernent, outre la grammaire et le lexique, certains « modes de constitution de l'information et d'organisation du discours » (Manessy 1994b, 11) et partant la dimension pragmatico-discursive. Or, « la manière de mettre en œuvre une langue [...] qui se trouve en quelque sorte transmuée (et non point pervertie) par l'émergence de schèmes cognitifs, de techniques d'expression, de modes d'énonciation » (Manessy 1994b, 225) n'a guère été explorée. Parmi ces phénomènes pragmatico-discursifs routines conversationnelles et marqueurs de discours, très sensibles au contact linguistique, méritent une attention particulière.

\section{Mots du discours et contact linguistique}

Formes morphosyntaxiquement libres qui remplissent des fonctions pragmaticocommunicatives quasiment universelles, les marqueurs du discours sont facilement transférés d'une langue à l'autre (Auer/Maschler 2016). Matras (2000), qui s'intéresse à une influence de la L2 sur la L1, cherche les raisons pour cette disponibilité à l'emprunt dans des facteurs communicatifs et cognitifs du bilinguisme individuel. Il constate que les marqueurs sont parmi les premiers éléments à être empruntés dans des situations de contact. Ils se trouvent en haut d'une échelle «d'empruntabilité » (scale of borrowability) et préparent des transferts subséquents, d'ordre morphosyntaxique. À la différence de Matras, nous nous intéressons à l'influence potentielle d'une L1, appartenant à l'adstrat africain, sur la L2, le français. Ce n'est donc pas l'emprunt, mais l'interférence qui caractérise ce type de situations. À l'exception de quelques travaux en didactique de langues qui examinent les marqueurs discursifs dans une perspective acquisitionnelle, le comportement des marqueurs et, en général, des mots du discours dans des situations définies par un apprentissage incomplet de la L2, n'a guère été analysé. Il ressort de ces études que l'usage des marqueurs est un indicateur fidèle de la compétence générale des locuteurs dans la L2. Ainsi, une étude sociolinguistique conduite auprès d'anglophones montréalais parlant le français comme L2 arrive-t-elle à la conclusion que «the least fluent, the least competent L2 speakers used almost no discourse markers , [...] the more successful L2 speakers were those who could control native discourse markers in a nativelike fashion » (Sankoff et al. 1997, 213). L'acquisition tardive des marqueurs dans une L2 s'explique par leur champ fonctionnel parfois très riche et nuancé. Comme ces petits mots «sont les signes de la maîtrise d'une langue seconde » (Charaudeau/Maingueneau 2002, 365), il n'est guère surprenant que leur usage en francophonie seconde diverge de celui en FH. Ici, 
interférences et traditions communicatives différentes favorisent une évolution autonome de ces formes et locutions. Aussi, les marqueurs de discours et, de façon générale, les mots du discours représentent-ils un domaine particulièrement fructueux pour les recherches sur les FA.

Les rares études consacrées aux marqueurs dans les FA montrent que l'on trouve, à côté d'emprunts aux langues de contact comme $k e$ en français populaire ivoirien (Abolou 2010), des formes du FH ayant subi un glissement dans leur sens pragmatico-discursif (Drescher 2015) ou une modification de leur fréquence. D'après notre connaissance, les routines conversationnelles, et plus précisément les emplois interjectifs de pardon, n'ont pas encore fait l'objet d'une analyse nuancée dans les FA, mis à part une note de Frey (2015) sur laquelle on reviendra plus tard. En revanche, certains dictionnaires et grammaires proposent une description des différents emplois de pardon qui nous permet de saisir son sens pragmatico-discursif. Il nous servira de cadre pour les analyses subséquentes. Précisons que si nous adoptons une perspective contrastive, nous ne visons pas une étude différentielle. Une meilleure connaissance des emplois de pardon en FH a surtout un effet de loupe et nous permettra de mieux identifier les spécificités des FA.

\section{Les emplois interjectifs de pardon}

Le nom masculin pardon a une étymologie religieuse. D'après le Petit Robert (2011, s.v.), il est dérivé du verbe pardonner, anciennement "perdoner (un pêché) 'remettre à qqn (la punition d'un pêché)' ». Le Grand Robert (en ligne, s.v.) distingue sous l'entrée pardon trois sens: 1. «action de pardonner»; 2. «indulgences accordées aux fidèles par l'Église »; et 3. «formule de politesse par laquelle on s'excuse de déranger ou d'interrompre quelqu'un, d'avoir à lui demander un service [...], de lui faire répéter une phrase qu'on a mal comprise [...], de le contredire... ou pour introduire une rectification ». C'est ce troisième sens qui ne concerne que les emplois interjectifs de pardon qui importe ici. Il comprend un emploi considéré comme familier où pardon apparaît dans une exclamation superlative et s'emploie en incise, comme mot emphatique. Et on mentionne aussi la formule pardon excuse !. Le Trésor de la langue française informatisé (TLFI en ligne, s.v.), plus riche en exemples que le Grand Robert, indique également trois sens et note en deuxième position les emplois de pardon «dans des tours ou formules avec atténuation du sens » où il serait usuel 1. "dans des formules de politesse employées en diverses circonstances », mais aussi 2. "pour faire répéter ce qu'on a mal entendu » et 3. "pour apporter une correction, une contradiction ». On relève également un emploi exclamatif, considéré ici comme populaire: «exclam. qui prévient l'étonnement de l'interlocuteur ou marque l'étonnement admiratif ». Dans l'exemple cité (provenant d'un roman de Simonin) pardon se combine avec le marqueur de discours polyvalent mais qui semble ajouter une touche d'emphase : Le projecteur n'éclairait de la harpiste que les pognes se baguenaudant sur les cordes; mais pardon rien que ça valait déjà le déplacement. En revanche, le Petit Robert (1994, s.v.) ne distingue que deux emplois différents : 1. «Tenir (une offense) pour non avenue, ne pas en garder de ressentiment, renoncer à en tirer vengeance »;2. "(Sens atténué) Juger avec indulgence, en trouvant des excuses, en minimisant la faute ». Pardon en tant qu'interjection est considéré comme une ellipse de la formule Je vous demande pardon. ${ }^{1}$ Parmi les grammaires, seuls Damourette/Pichon (1968) consacrent un paragraphe entier (§ 758) à ses emplois interjectifs. Après avoir souligné que pardon sert à « exprimer qu'on implore, et non qu'on accorde un pardon », ils poursuivent : "Mais il peut s'atténuer beaucoup sémantiquement et ne plus être qu'une banale formule de politesse » (Damourette/Pichon 1968, 455). Cette formule de politesse connaît des fonctions variées : "Pardon est, en somme, dans la langue moderne, une formule courante de politesse, qui s'emploie dans certaines circonstances où il n'y a, à vrai dire, pas d'offense réelle dont s'excuser, mais où l'omission de pardon serait 
impolie [...]. Les répons usuels sont : 'Je vous en prie ; mais faites donc, faites donc, faites' [...]. Dans certains milieux, il passe même pour malséant d'employer [...] si, et on le remplace régulièrement par pardon, comme pour s'excuser de contredire son interlocuteur » (Damourette/Pichon 1968, 455).

De par son origine dans un contexte religieux, social et moral déterminé, on peut présumer que pardon fait partie des "catégories culturelles » au sens D'Andrade (1984). Son contenu notionnel premier est enraciné dans la pensée chrétienne et marqué par la culture occidentale. Même dans ses emplois banalisés, cette formule de politesse garde des connotations religieuses et sociales qui ne sont pas forcément universelles. Son appartenance à une culture donnée semble s'opposer à sa simple transposition dans un autre contexte culturel dont pourraient témoigner les emplois saillants rencontrés dans les données. Certains de ces emplois spécifiques dans les FA ne sont pas restés inaperçus. Ainsi, l'inventaire de l'équipe IFA $\left({ }^{3} 2004,281\right)$ prévoit-il une entrée pour pardon : «interj. BE., B.F., C.I., NIG. (oral), SEN., TO., ZA. (dial., oral) (Accompagnant une requête). S'il te plaît, je t'en prie, je t'en supplie. 'Donne-moi $10 \mathrm{Ka}$, pardon!' ». Cet usage particulier où pardon remplace d'autres formules de politesse est observé dans sept pays francophones allant du Sénégal au nord jusqu'à la RDC au sud. Le Cameroun, d'où proviennent la majorité de nos exemples, ne figure pas dans cette liste. En revanche, le dictionnaire électronique Wiktionary le considère comme spécifique à la Côte d'Ivoire : "(Ivoirisme) Formule de politesse pour demander un service ou atténuer une demande; souvent équivalent de $s$ 'il vous plait. Donne-moi un peu d'eau à boire pardon. Pardon mon frère faut pas te fâcher, viens t'asseoir on va causer, ça va aller. " ${ }^{2}$ Ces deux sources indiquent cependant que l'extension du champ fonctionnel de pardon ne se limite pas à un pays, ni même à une sous-région de la francophonie africaine. Elles restent cependant vagues quant aux différentes fonctions pragmatico-discursives de pardon dans les FA.

\section{Analyses}

L'analyse d'un corpus constitué d'interactions orales spontanées, provenant des français camerounais et burkinabé, permettra de nuancer davantage les valeurs pragmaticodiscursives de pardon et, de manière générale, d'illustrer l'originalité et la force innovatrice des FA sur le plan pragmatico-discursif. Les données proviennent de trois corpus partiels. Il s'agît d'abord d'interactions entre des soi-disant docteurs, qui sont en fait de vendeurs de médicaments sans formation académique en médecine, et des passagers dans des cars interurbains au Cameroun et au Burkina Faso. ${ }^{3}$ Un troisième corpus partiel est constitué de tribunes radiophoniques vouées aux problèmes de santé (Drescher 2014). ${ }^{4}$ En tout 80 occurrences de pardon ont été retenues pour cette étude, dont 69 issues de données camerounaises et $11 \mathrm{du}$ corpus burkinabé. ${ }^{5}$ L'analyse part des différents contextes séquentiels de pardon en tenant compte de sa position initiative ou réactive et de sa place au sein d'un tour de parole (début, milieu, fin). La plupart des occurrences de pardon se trouvent au milieu du tour de parole alors que la position initiale est moins fréquente. Comme le corpus ne contient qu'une occurrence avec pardon à la fin d'une intervention, ce type sera écarté par la suite. En revanche, il peut constituer à lui seul un tour de parole ou être accompagné d'un terme d'adresse. Soulignons aussi que les données relèvent d'un français oral, spontané et 'endogénisé'. 6

\section{1 pardon comme intervention réactive}

Quand pardon constitue à lui seul un tour de parole, il apparaît toujours en position réactive, c'est-à-dire comme réaction à une intervention préalable. Dans l'exemple suivant, le locuteur l'emploie pour demander à son interlocuteur de répéter une question. Pardon 
déclenche une reprise mot-à-mot et fait partie d'une «auto-reformulation hétérodéclenchée » (Gülich/Kotschi 1987).

(1)

NAR : quelle est la capacité (.) ou la quantité d'eau (-) minimale (.) que l'on doit con/

(.) doit consommer pour vingt-quatre heures,

PAS : un litre et demi

NAR : pardon,

PAS : un litre et demi

Cette fonction a déjà été relevée par les dictionnaires qui notent que pardon sert à «faire répéter ce qu'on a mal entendu » (TLFI, s.v.). Le français camerounais la partage donc avec le FH. Le locuteur interrompt son interlocuteur à l'aide de pardon et initie une séquence latérale permettant de résoudre un problème de compréhension qui risquerait autrement de mettre en danger l'interaction. Il s'agît là d'une fonction clairement discursive. Précisons que pardon se prête aussi à un emploi stratégique où le locuteur fait semblant de ne pas avoir entendu.

\section{2 pardon à l'intérieur d'un tour de parole}

On peut différencier les occurrences où pardon se trouve à l'intérieur d'un tour de parole à l'aide de critères structurels et formels. Ils ont trait à sa position au sein du tour de parole et aux formes qui l'accompagnent, par exemple des termes d'adresse (tonton, papa, patriarche, docteur, monsieur) ou d'autres formules de politesse (notamment s'il vous plaît). Selon sa position, on peut distinguer des emplois où pardon indique une autocorrection auto-déclenchée, signale une interruption ou marque le début du discours rapporté.

\subsection{1 « pardon » marqueur d'auto-correction auto-déclenchée}

Avec ce procédé le locuteur revient sur (une partie de) son énoncé en lui substituant une nouvelle formulation ce qui donne une séquence tripartite constituée par une première formulation, un marqueur et une reprise/correction (Gülich/Kotschi 1987). C'est pardon qui sert ici comme marqueur de correction. Dans (2), le locuteur corrige un lapsus en remplaçant le syntagme une nuit par une fois. L'exemple (3) portant sur une confusion des côtés gauche et droite, est intéressant parce que pardon n'est pas placé entre les deux segments, mais vient seulement après la reprise (côté droite) en précisant rétroactivement qu'il s'agît d'une correction qui est ensuite ratifiée par l'interlocuteur.

(2)

c'est pourquoi on appelle ça le cataplasme tissulo cellulaire (.) vous reprenez ça une nuit pardon une fois (.) deux fois (.) trois fois ça fait neuf jours

(3)

A : j'ai euh : pendant ma période de fécondité j'ai constamment $\mathrm{mAl}$ au bas-ventre côté gauche côté droite pardon

$\mathrm{DE}: \mathrm{h}=\mathrm{hm}$

Cet emploi est également connu en FH. Les dictionnaires précisent que pardon est utilisé " pour introduire une rectification » (Grand Robert) ou «pour apporter une correction, une contradiction » (TLFI). À la différence du TLFI, il nous semble judicieux de distinguer la correction de la contradiction, à cause notamment des répercussions que cette dernière peut avoir sur la relation interpersonnelle. ${ }^{7}$ L'exemple (4) montre que pardon peut être interprété ou comme un marqueur de correction ou comme un moyen pour gagner l'attention du public. 
(4) les papas dans la voiture ci pardon (.) les hommes écoutez le conseil on ne sait jamais

Dans le premier cas, papas - terme de respect courant en FA qui n'exprime pas forcément une relation de parenté, mais peut désigner tout homme d'un certain âge - serait remplacé par hommes, terme plus neutre et partant plus général qui inclut tous les individus masculins au-delà de l'âge de l'adolescence. La reprise de papas par hommes marquerait alors une extension des destinataires visés par ce message. Dans le deuxième cas, pardon serait équivalent de s'il vous plaît ou de la forme écoutez et c'est alors la fonction appellative qui prime.

\subsection{2 « pardon » marqueur d'interruption}

Pardon peut indiquer aussi une auto-interruption provoquée par le contexte. Le locuteur suspend alors momentanément l'interaction principale pour ouvrir une séquence latérale. Dans (5), cette coupure, causée par l'arrivée d'un nouveau participant, concerne le cadre de participation tandis qu'en (6), c'est l'urgence d'exécuter une activité non-verbale (fermer la vitre) qui justifie l'interruption.

(5)

NDI : madame ton corps devient fine [sic] (.) ton ventre plat et les fesses aromatiséespardon monsieur bonjour

PAM : bonjour

NDI : je dis hein monsieur,

(6)

ça va permettre à ce qu'il y aura l'évacuation de l'eau sale dans votre organisme-(-) pardon essayez de fermer la vitre là derrière $\mathrm{i}(1)$ y a les enfants là-bas (.) grand sœur pousse un peu la vitre là

Cet emploi, proche de celui où pardon constitue à lui seul une intervention réactive (cf. 5.1), s'en distingue cependant en deux points : c'est le locuteur qui s'interrompt lui-même et l'interruption est causée par un événement survenu dans le contexte de l'interaction et non pas par un problème de compréhension.

\subsection{3 « pardon » balise du discours rapporté}

Le discours rapporté apporte une rupture du dispositif énonciatif qui peut être signalée par des changements prosodiques, des verba dicendi comme dire ou encore des marqueurs. Bien qu'il n'y ait pas de forme spécifiée exclusivement dans l'introduction du discours rapporté, certains marqueurs y apparaissent de façon privilégiée. Pardon semble être parmi les mots qui servent comme balises du discours rapporté. Faisant partie du discours cité, il se trouve à la charnière du discours assumé par le locuteur et du discours rapporté. Dans (7), le verbe dire contribue à indiquer ce changement énonciatif tandis que dans (8), où pardon est précédé du terme d'adresse chauffeur, c'est le verbe crier.

(7)

elle compte le plafond (.) elle tue les moustiques- (.) elle dit à l'homme que quoi pardon fais vite tu te lèves tu sors (.) je suis trop fatiguée

(8)

c'est là où des gens crient au scandale (.) chauffeur pardon gare un peu ton bus

Dans l'extrait (9), pardon fait partie d'une construction subordonnée articulée par que qui introduit le discours indirect. La demande indirecte s'explique par le format particulier de l'émission où l'auditrice s'adresse à l'animateur de la tribune radiophonique qui transmet 
ensuite sa requête au tradipracticien (docteur) également présent dans le studio. Qu'elle soit directe ou indirecte, pardon contribue à marquer une rupture énonciative.

(9)

je voulais demander au docteur que pardon s'il pouvait me donner une recEtte pour qu'elle ait/ pour qu'elle puisse avoir l'équilibre de rester debout seule

En même temps, pardon contient ici une nuance appellative qui le rapproche de s'il vous plaît. Cette interférence, courante dans les FA comme l'indique déjà son entrée dans l'IFA, se remarque également dans les usages suivants où pardon s'accompagne d'un terme d'adresse.

\subsection{4 « pardon » suivi d'un terme d'adresse}

Le désir d'attirer l'attention de l'interlocuteur est primordial quand pardon précède un terme d'adresse. Ce dernier le supporte dans sa fonction conative tout en spécifiant le destinataire du message. Pardon est alors équivalent à s'il vous plaît comme dans (10).

\section{(10)}

on ne met pas l'eau plein au départ (.) mets de l'eau à moitié (.) pardon papa mettez moi l'eau à moitié dans le source tangui ${ }^{8}$

Dans l'extrait (11), la fonction de pardon qui s'associe à la locution s'il vous plaît, est ambiguë.

(11)

s'il vous plaît pardon patriarche (.) je voulais que vous parlez aux jeunes ${ }^{9}$

D'un côté, pardon semble garder sa valeur première d'excuse rituelle en accompagnant une interpellation qui adoucit une intrusion dans le territoire de l'autre. D'un autre côté, il se trouve en quelque sorte dans le halo de s'il vous plaît qui déteint sur son sens pragmaticodiscursif. Bien que reparties sur deux formes, atténuation et interpellation se combinent dans cet exemple. Il est tout à fait probable que c'est dans ces contextes que pardon a incorporé certains traits de s'il vous plaît au point de pouvoir s'y substituer. Pour mieux comprendre cette fonction, attestée par les extraits suivants, nous commençons par rappeler les emplois de s'il vous plaît tels que décrits dans les dictionnaires et grammaires du FH. Cela permet de voir aussi si, à côté de facteurs externes, écologiques, il existe des facteurs internes favorisant une telle évolution.

\subsection{5 « pardon » synonyme de « s'il vous plaît »}

Selon le Petit Robert (2011, s.v.), s'il vous plaît est utilisé « dans une demande, un conseil, un ordre $»-$ contextes où la locution serait synonyme de je vous prie. ${ }^{10}$ En outre, elle est employée «Pour souligner un avertissement », "Pour attirer l'attention sur ce qu'on vient de dire, de nommer ». Grevisse $\left({ }^{10} 1975,1064\right.$ s. $)$ effleure certains usages de s'il vous plaît dans un paragraphe consacré à voici et voilà. La locution peut remplacer les présentatifs « spécialement quand on veut attirer l'attention de la personne à qui on présente quelque chose ». Avec de nombreux exemples à l'appui, il précise que s'il vous plaît « se dit pour demander quelque chose à quelqu'un ou pour le prier de faire quelque chose » et il « se dit aussi pour recommander avec énergie ou pour souligner ce qu'on dit ». ${ }^{11} \mathrm{Si}$ ces fonctions n'ont que peu en commun avec celles de pardon, il existe néanmoins un contexte où $s$ 'il vous plaît peut s'y substituer, à savoir pour faire répéter un énoncé (cf. 5.1). Cet emploi est pourtant critiqué par Grevisse $\left({ }^{10} 1975,1064 \mathrm{~s}\right.$.) qui recommande: «Pour prier un interlocuteur de répéter ce que vous n'avez pas entendu clairement ne dites pas : s'il vous plaît? Dites : plaît-il ? ou comment? [...] ou pardon? (hein ? est du langage très familier ; - quoi ? est vulgaire ou impoli) ». En résumé il apparaît que, si les deux expressions 
montrent certaines ressemblances en FH, elles sont loin d'être synonymes. ${ }^{12}$ Or, on observe dans les données une extension des usages de pardon qui s'annexe bon nombre de fonctions de s'il vous plaît. L'interchangeabilité des deux formes semble néanmoins se limiter à certains contextes pas toujours faciles à distinguer, car les modifications du sens pragmatico-discursif de pardon sont subtiles, parfois graduelles et largement dépendantes du contexte. Cela vaut notamment pour les fonctions argumentative (cf. 5.2.6) et conative. Dans ce deuxième cas, illustré par les extraits suivants, pardon apparaît comme un synonyme de $s$ 'il vous plaît servant à interpeller ou à focaliser l'attention de quelqu'un.

Examinons d'abord les exemples (12) et (13) où pardon et s'il vous plaît sont cooccurrents. On peut se demander si pardon y garde son sens premier d'atténuateur ou s'il sert plutôt à moduler voire à renforcer s'il vous plaît. Le fait que dans (12) les deux expressions encadrent une reprise paraphrastique et que dans (13) elles semblent avoir une fonction interpellative, nous fait pencher vers l'hypothèse d'une valeur avant tout emphatique.

(12)

pas à tout le monde (.) pardon pas à tout le monde s'il vous plaît

(13)

mais s'il vous plaît madame de la question (.) pardon posez la question rapidement

Les exemples où pardon est synonyme de s'il vous plaît sont nombreux. Pardon accompagne souvent un acte de langage directif, en général une requête du locuteur. Il souligne sa valeur illocutoire en ajoutant une touche d'emphase. À l'exception de (18), tous les exemples comprennent des verbes à l'impératif, le plus souvent à la deuxième personne du pluriel, qui explicitent le caractère injonctif de l'énoncé (déchirez, faites, levez, fais, etc.).

sortez le sachet de thé vert (.) ne déchirez pas pardon (.) jetez à l'intérieur de l'eau chaude

ceux qui ont pris les produits là (.) pardon faites-moi votre argent ${ }^{13}$

(16)

si à bord de ce bus il y a des personnes qui ont ce problème- (.) pardon levez la main

donnez-moi une minute pardon donnez-moi une minute

Dans (18), et à la différence des autres extraits, la requête est formulée de manière indirecte à l'aide de la phrase déclarative vous me faites signe. Comme dans les exemples précédents, pardon segmente néanmoins l'intervention.

(18)

pour ceux qui sont intéressés vous me faites signe pardon on est encore là

Précédé ou suivi d'une courte pause, pardon accentue une structure bipartite dont il est le pivot et contribue ainsi à détacher les parties de l'intervention. On retrouve un contour prosodique semblable dans les emplois argumentatifs de pardon.

\subsection{6 « pardon » et l'articulation argumentative}

Les dictionnaires consultés mentionnent pour le FH un emploi que l'on pourrait qualifier d'argumentatif. Selon le TLFI, on utilise pardon pour apporter une contradiction. Petit et Grand Robert accordent davantage d'importance au sens premier du mot pardon en insistent sur le fait qu'il sert à s'excuser de contredire quelqu'un. En s'excusant à l'avance le locuteur préviendrait une menace pour la face de l'autre et partant pour la relation interpersonnelle. Pardon serait donc avant tout un atténuateur de contradiction. Cette 
interprétation est corroborée par Damourette/Pichon (1968, 455) qui affirment: «Dans certains milieux, il passe même pour malséant d'employer [...] $s i$, et on le remplace régulièrement par pardon, comme pour s'excuser de contredire son interlocuteur ». Mais les auteurs prennent soin de nuancer que le locuteur fait semblant de s'excuser. ${ }^{14}$ De nos jours, cette 'excuse' semble complètement banalisée de sorte qu'elle contribue moins à atténuer qu'à renforcer la valeur contre-argumentative de l'énoncé. Dans ces emplois routinisés pardon ne minimise guère l'offense potentielle émanant de la contradiction, bien au contraire, il ne la rend que plus visible. À l'origine un marqueur d'atténuation, pardon semble signaler désormais de façon conventionnelle la contradiction.

Il reste qu'à l'oral spontané les enchaînements - qu'ils soient thématiques ou argumentatifs - sont souvent flous, car la structuration du discours se fait plutôt sur le mode agrégatif. De plus, le recours au contexte et à l'implicite joue un rôle nettement plus important qu'à l'écrit. Comme les liens entre les énoncés ne sont que rarement explicités, l'argumentation peut paraître décousue ou afficher un manque de cohérence. Voilà pourquoi il n'est pas évident de distinguer d'abord emplois structurants et argumentatifs, et de déterminer ensuite le mouvement argumentatif auquel participe pardon. Même si les transitions sont plutôt fluides, se dessine un contexte particulier où pardon met en relief la structure bipartite d'une phrase conditionnelle.

tu crois que ça va chez toi' (.) pardon (.) le remède là' vas essayer

prends un vase, (.) mets-toi quelque part et puis fais tes besoins (.) quand tu as fini là, pardon, prends le courage de regarder ce qui est sorti de ton ventre

(21)

le vieux il est couché (.) il peut pas se lever (.) pardon donne lui ça

même si vous êtes tous pareils (.) pardon excusez moi, les cinq doigts de la main n'ont pas la même taille- (.)

Parfois, le mouvement argumentatif est complexe et pardon semble baliser le recours à un constituant implicite comme dans les exemples (19) et (22) que l'on pourrait compléter par il se trompe/vous vous trompez. De plus, dans (22) la formule excusez-moi se trouve dans le cotexte immédiat de pardon et remotive en quelque sorte son sens premier. Dans tous les extraits, pardon contribue à introduire une certaine bipolarité. Sa valeur contrastive pourrait être dérivée d'une de ses fonctions dialogiques, à savoir signaler une contradiction, même si un tel emploi, prédominant en FH à en croire les dictionnaires et grammaires, n'est attesté que deux fois dans nos données (cf. 5.3). Bien que ces différentes instructions argumentatives restent à creuser davantage, les analyses montrent que pardon occupe une place charnière à l'intérieur de l'énoncé. Par ailleurs, une analyse systématique des contours prosodiques de pardon reste à faire. Elle contribuera certainement à nuancer davantage ses fonctions.

Si les emplois de pardon au sein d'un tour de parole abondent, ceux au début sont bien moins nombreux et leurs fonctions semblent se recouper avec celles déjà relevées.

\section{3 pardon au début du tour de parole}

Au début d'un tour de parole pardon signale ou une interruption ou une contradiction. En (23), il est un marqueur d'interruption à l'aide duquel le locuteur essaie de mettre un terme aux rires et de regagner l'attention du public. Pardon a une valeur appellative et équivaut à s'il vous plaît. 
(23)

PAF8 : c'est sucré

PAS : ((rire))

ROB : pardon goûtez à la maman là derrière- $(.)^{15}$ vous voilà parce que le plus souvent les gens voient comme ça ils disent que ils pensent que c'est l'huile d'arachide

L'extrait (24) recèle une des deux occurrences du corpus où pardon est un marqueur de contradiction. Il est tiré d'une séquence de marchandage. Vu le prix trop bas, proposé par le client, le docteur refuse de continuer la transaction.

(24)

ROB : j'ai dit mi(lle) francs (.) mi(lle) francs cinq personnes-

PAF : $\quad$ cinq cent

ROB : pardon je préfère qu'on laisse

Il est clair que le type d'interaction a un impact sur la fréquence des différents emplois de pardon. On peut présumer que le nombre de marqueurs de contradiction est plus élevé dans des débats ou des controverses que dans les consultations radiophoniques ou les interactions commerciales exploitées ici. ${ }^{16}$

\section{En guise de conclusion : L'appropriation entre langue(s) et culture(s)}

Les analyses ont mis en évidence que pardon a considérablement élargi son champ fonctionnel. Au-delà des emplois partagés avec le $\mathrm{FH}$, les français camerounais et burkinabé ont conventionnalisé de nouvelles fonctions. Par surcroît, pardon a emprunté des traits à d'autres routines conversationnelles, notamment à la locution s'il vous plaît qu'il supplante dans certains contextes. Il est intéressant de noter que ces mêmes formes connaissent des modifications dans les français est-africains en évoluant toutefois dans des directions différentes. Dans une brève étude de trois lexies du français utilisé en Afrique francophone et au Kenya, Frey (2015) attire notre attention sur des emplois où pardon prend le sens de 'désolé'. Il y voit une influence du mot swahili pole qui se traduit et par 'excusez-moi' et par 'désolé' de sorte que «pardon est saisi dans le champ sémantique africain de pole qui exprime la compassion et la sympathie » (Frey 2015, 98). Malgré une base empirique peu abondante, ${ }^{17}$ Frey n'hésite pas à conclure que ces nouvelles fonctions révèleraient « des représentations socioculturelles africaines » (Frey 2015, 105) et à imputer cette évolution à d'autres conventions de politesse : "pardon développe donc un champ lexico-sémantique particulier lié à une attitude sociale et des règles de courtoisie différentes » (Frey 2015, 98).

Quoique sa démarche nous paraisse quelque peu hâtive, il ressort des observations de Frey qu'une même 'construction', comprise comme unité de forme et de fonction au sens de la grammaire de construction, peut, selon le milieu et notamment les langues de contact, évoluer dans des directions différentes, voire opposées. Et il ne fait aucun doute que le contenu notionnel fortement culturel de pardon le prédispose à une telle appropriation. L'argumentation de Frey soulève cependant un certain nombre de problèmes. Mise à part le manque d'une base empirique plus étoffée et surtout plus homogène qui permettrait de mieux contrôler les multiples variables en question (L1 des locuteurs et leur compétence en français, langues de contact respectives, situation de communication, etc.), on est surpris par l'influence présumée du swahili sur des français qui ne sont point en contact avec cette langue véhiculaire est-africaine. Il est peu probable que ses conventions sociales et discursives puissent avoir un impact sur le français au Cameroun, à moins de considérer le swahili comme représentatif des langues africaines, ce qui semble problématique au regard de la grande diversité ethno-linguistique du continent. Et même si l'Afrique subsaharienne formait une communauté discursive relativement homogène, ce serait abusif de conclure de 
l'emploi particulier d'un seul terme à des règles de courtoisie différentes. On peut contester à juste titre la prétention universaliste de la théorie de la politesse dans le sillage de Brown/Levinson $\left({ }^{2} 1987\right)$ et lui reprocher sa valorisation de l'individu ancrée dans la tradition occidentale comme le fait Mulo Farenkia (2008) qui insiste sur l'importance de la collectivité et l'adhésion au groupe, constitutives des sociétés africaines. Conclure du simple fait que pardon acquiert de nouvelles fonctions à « une vision différente des relations interpersonnelles » (Frey 2015, 98) et valider cette hypothèse par le recours à la «psychologie africaine » (Frey 2015, 98) suscite pourtant des réserves. Rappelons quand même que le FH ne manque pas de mot pour exprimer la compassion ou le regret. Il dispose justement de l'adjectif désolé. Seulement, et à la différence du swahili, les deux sens sont repartis sur les deux lexies désolé et pardon. Mieux que de spéculer sur l'altérité des cultures vaut donc de creuser davantage l'idée d'une influence des langues de contact. Comme Frey le laisse entendre lui-même il s'agît vraisemblablement d'une interférence avec le terme pole qui couvre justement les deux sens. Et dans les français burkinabé et camerounais, une interférence semble également être à l'origine des nouveaux sens pragmatico-discursifs de pardon. Ainsi, plusieurs langues bamilékées parlées dans l'ouest du Cameroun, n'ont qu'un seul mot là où le français dispose de pardon et de s'il vous plaît ce qui pourrait expliquer la confusion observable dans les données. ${ }^{18}$ Rappelons aussi que, de façon générale, les mots du discours posent problème à des locuteurs non-natifs. La difficulté au moment de l'apprentissage semble due au sens pragmatico-discursif à la fois souple et contextuel de ces mots et locutions qui pourrait faciliter leur interchangeabilité avec d'autres formes appartenant à la même catégorie fonctionnelle et donner ainsi naissance à de nouvelles fonctions. Cette 'élasticité' sémantico-pragmatique explique aussi pourquoi, selon les langues de contact, l'évolution peut prendre des directions différentes. Enfin, il n'est pas à exclure non plus qu'un manque de familiarité avec les conventions de politesse de la culture d'origine puisse favoriser cette tendance sans pourtant être le seul facteur responsable.

Nos analyses mettent en relief l'importance du niveau pragmatico-discursif pour une différenciation interne de l'espace variationnel africain. Cette dernière se heurte à de nombreux problèmes qui se posent d'abord à l'échelle de la francophonie africaine et ensuite au sein de ce que l'on pourrait appeler les différents 'français nationaux'. Le débat, déjà ancien, tourne autour de la question de savoir s'il existe un français ou des français africains. Malgré certains facteurs uniformisateurs, l'émergence de 'variétés' nationales ou du moins caractéristiques d'une sous-région - ne semble plus faire de doute aujourd'hui alors que la question de savoir en quoi ces 'variétés' diffèrent au juste demeure toujours sans réponse satisfaisante. ${ }^{19}$ Ici, une analyse détaillée des phénomènes de discours pourrait fournir des indices supplémentaires. S'ils participent partout à la dynamique du français, il semble que routines conversationnelles et marqueurs de discours peuvent connaître des évolutions différentes selon le paysage linguistique respectif. L'exemple de pardon qui adopte des sens différents dans les français ouest- et est-africains est instructif à cet égard. Pour valider une telle hypothèse, il faudrait cependant compléter notre approche, située au croisement des théories de la variation et de la pragmatique par une démarche comparative qui intègre les langues de l'adstrat et leurs formes équivalentes.

\section{Références bibliographiques}

C. R. Abolou. Des marqueurs 'ke' et 'non' en français populaire d'Abidjan : stratégies discursives et modélisation. Le français en Afrique 25, 325-342 (2010)

P. Auer, Y. Maschler. The family of NU and NA across the languages of Europe and beyond: Structure, function, and history. In P. Auer, Y. Maschler (edd.). NU/NA. A Family of Discourse Markers across the Languages of Europe and Beyond, Berlin/Boston, de Gruyter, 1-47 (2016)

P. Brown, S. C. Levinson. Politeness. Cambridge etc., Cambridge University Press $\left({ }^{2} 1987\right)$ 
L.-J. Calvet. Les mutations du français. Une approche écolinguistique, Le français moderne 68/1, 63$78(2000)$

P. Charaudeau, D. Maingueneau. Dictionnaire d'analyse du discours, Paris, Seuil (2002)

J. Damourette, É. Pichon. Des mots à la pensée, Paris, Éditions d'Artrey, tome II (1968)

R. D'Andrade. Cultural meaning systems. In : R. A. Shweder, R. A. LeVine (edd.). Culture theory. Essays on mind, self and emotion, Cambridge, Cambridge University Press, 88-119 (1984)

M. Drescher. Le Cameroun. In : U. Reutner (ed.). Manuel des francophonies, Berlin, de Gruyter, 431457 (2017)

M. Drescher. Hybridized Discourse Markers in Cameroonian French? The Example of 'déjà'. In : T. Stolz, I. Warnke (edd.). Papers of the 1st International Conference on Colonial and Postcolonial Linguistics (Bremen, September 3-8, 2013), Berlin, de Gruyter, 79-100 (2015)

M. Drescher. La dimension pragmatico-discursive du français en contact. L'exemple des consultations à la radio camerounaise, Journal of Language Contact 7, 62-92 (2014) http://booksandjournals.brillonline.com/content/journals/19552629/7/1

M. Drescher, I. Neumann-Holzschuh. Les variétés non-hexagonales du français et la syntaxe de l'oral. Première approche. In : M. Drescher, I. Neumann-Holzschuh (edd.). La syntaxe de l'oral dans les variétés non-hexagonales du français, Tübingen, Stauffenburg, 9-35 (2010)

Équipe IFA. Inventaire des particularités lexicales du français en Afrique Noire, Vanves, EDICEF/AUF ( ${ }^{3} 2004$ [1983])

C. Frey. De l'Afrique francophone au Kenya. Saisir le français entre les langues et les cultures avec 'pardon', 'les trois pierres' et 's'asseoir'. In : P. Blumenthal (ed.). Dynamique des français africains : entre le culturel et le linguistique, Frankfurt/Main, Peter Lang, 95-107 (2015)

Grand Robert (en ligne) (consulté le 21/06/2017) http://gr.bvdep.com/robert.asp

M. Grevisse. Le bon usage, Bruxelles, Duculot $\left({ }^{10} 1975\right)$

M. Grevisse, A. Goosse. Le bon usage, Bruxelles, de Boeck/Duculot $\left({ }^{14} 2008\right)$

E. Gülich, T. Kotschi. Les actes de reformulation dans la conversation 'La Dame de Caluire'. In : P. Bange (ed.), L'analyse des interaction verbales. La Dame de Caluire : une consultation, Bern etc., Peter Lang, 15-81 (1987)

G. Manessy. Pratique du français en Afrique noire francophone, Langue française 104, 11-19 (1994a)

G. Manessy. Le français en Afrique Noire. Mythe, stratégies, pratiques, Paris, L'Harmattan (1994b)

Y. Matras. Fusion and the cognitive basis for bilingual discourse markers, International Journal of Bilingualism 4.4, 505-528 (2000)

B. Mulo Farenkia (ed.). De la politesse linguistique au Cameroun, Frankfurt/Main, Peter Lang (2008)

Petit Robert. Le Petit Robert. Dictionnaire alphabétique et analogique de la langue française. Paris, Le Robert (1994 et 2011)

K. Sachs, C. Villatte. Enzyklopädisches Französisch-Deutsches und Deutsch-Französisches Wörterbuch, Berlin-Schöneberg, Langenscheidtsche Verlagsbuchhandlung (1911)

G. Sankoff, P. Thibault, N. Nagy, H. Blondeau, M.-O. Fonollosa, L. Gagnon. Variation in the use of discourse markers in a language contact situation, Language Variation and Change 9, 191-217 (1997)

D. Schiffrin. Discourse markers, Cambridge, Cambridge University Press $\left(1987 /^{2} 1996\right)$

M. Selting et al. Gesprächsanalytisches Transkriptionssystem 2 (GAT 2), Gesprächsforschung Online-Zeitschrift zur verbalen Interaktion 10, 353-402 (2009)

Trésor de la langue française informatisé (TLFI) : http://www.atilf.fr/tlfi (consulté le 20/06/2017).

1 L'entrée correspondante du Petit Robert (2011, s.v.) développe cependant davantage les emplois discursifs de pardon: "Je vous demande pardon, ou ElLIPT Pardon, ou Mille pardons: formule de politesse par laquelle on s'excuse (de déranger qqn, d'avoir à lui demander un service, de lui faire répéter une phrase qu'on a mal comprise [...], de le contredire ou qui sert à introduire une rectification $»$.

2 cf. https://fr.wiktionary.org/wiki/pardon, consulté le 23/06/17.

3 Le corpus camerounais a été gracieusement mis à ma disposition par Liliane Ngawa. Le corpus burkinabé, beaucoup plus petit, a été constitué par Daouda Traoré que je remercie également de son autorisation à utiliser ses données.

4 Le corpus camerounais comporte environ 167.000 mots (corpus partiel Ngawa environ 122.000 mots et corpus partiel Drescher environ 45.900 mots); le corpus burkinabé se compose d'environ 20.000 mots. 
Même si pardon peut être considéré comme une ellipse de demander pardon, les quelques occurrences de cette collocation ne seront pas traitées ici.

Nous utilisons les conventions de transcription de GAT 2 qui indiquent les phénomènes suivants : pauses très courte (.) et courte $(-)$; intonation très montante (?), montante ('), en suspens (-), descendante (,); accent (trËs) ; activités non verbales ((rire)) ; articulation relâchée (i(1)) (Selting et al. 2009).

Bien que leur statut interactionnel soit différent, correction et contradiction se ressemblent dans leur retour sur une entité discursive préalable. Alors que, dans le cas d'une contradiction, le locuteur récuse une intervention de l'interlocuteur, la correction peut porter et sur une formulation du locuteur (auto-correction) et sur un énoncé de l'interlocuteur (hétérocorrection). Ce dernier cas, plus dangereux pour la face, peut se confondre avec une contradiction.

La source tangui est une marque camerounaise d'eau minérale.

Le terme d'adresse patriarche exprime le respect pour un homme âgé.

La variante $s$ 'il te plaît, usuelle lorsqu'on tutoie l'interlocuteur, est totalement absente de nos données. La locution est donc complètement figée en FA et les pronoms personnels tu et vous ne sont plus perçus comme tels.

Le traitement de s'il vous plaît dans la dernière édition (Grevisse/Goosse ${ }^{14} 2008$, 1418s.) diffère légèrement de celui en 1975. Listée parmi les mots-phrases, la locution est considérée maintenant «comme élément incident, pour accompagner une demande» ou "pour recommander avec énergie ou pour souligner ce qu'on dit». Le fait que «cette expression est utilisée couramment en présentant quelque chose à quelqu'un ou pour le prier de répéter ce qu'il a dit » est interprété comme un belgicisme. On ajoute cependant que cet emploi n'est pas inconnu en France non plus.

Je remercie un des relecteurs anonymes d'avoir attiré mon attention sur le fait que, si l'on insère l'analyse dans une perspective écologique plus large sur le changement linguistique, pardon et s'il vous plaît partagent des valeurs d'emphase et de focalisation qui pourraient servir de base au processus analogique.

Le verbe faire a ici le sens de donner.

À cet égard le fait qu'un dictionnaire français - allemand du début du siècle dernier insiste déjà sur cette valeur argumentative est révélateur: "entschuldigen Sie (auch beim Aussprechen einer anderen Ansicht) » (Sachs/Villatte 1911, s.v.) (excusez (aussi en prononçant une opinion différente; traduction de l'auteure).

Le sens ici est bien sûr celui de la construction causative faire goûter. Maman est un terme d'adresse courant pour une femme d'âge mûr.

De manière générale, notre base empirique n'est ni suffisamment large, ni assez diversifiée pour décider si les emplois relevés se limitent à certains types d'interaction ou s'ils sont typiques des FA.

Élusif quant à la constitution et à la taille de son corpus, l'auteur présente au total sept exemples qui sont de surcroît très hétérogènes, car produits par des locuteurs anglophones et francophones venant de pays différents tels le Kenya, la France, le Burundi, le Congo et même le Cameroun. Il s'agît d'énoncés glanés au vol dans des conversations ou relevés dans des textos et dont seulement deux contiennent pardon alors que les autres comportent des formes considérées comme synonymes (sorry, patience et pole).

En medumba, une des langues bamilékées, c'est le mot netfho qui correspond en français, selon les contextes, à pardon ou à s'il vous plaitt (communication personnelle de Liliane Ngawa). En revanche, en dioula, langue véhiculaire en Côte d'Ivoire où cet emploi est également attesté au point d'être considéré comme un ivoirisme, il existe bien deux mots différents: pardon se dit sabari et l'équivalent de s'il vous plait est hakèto (communication personnelle d'Adama Drabo).

La même question se pose au niveau de ces 'français nationaux' camerounais, ivoiriens, sénégalais, etc. qui connaissent à leur tour une différenciation interne aboutissant éventuellement à la naissance de sociolectes (Drescher 2017). 